\title{
El uso de Inhibidores de la bomba de protones puede aumentar el riesgo de fracturas óseas
}

\author{
The use of inhibitors of the proton pump can increase the risk of bone fractures
}

\section{Objetivos}

El objetivo de este meta análisis fue evaluar la asociación entre el uso de inhibidores de la bomba de protones o de antagonistas de los receptores histamínicos $\mathrm{H} 2$ y fracturas óseas. El resultado primario fue analizar la asociación con fractura de cadera. Los resultados secundarios incluyeron cualquier sitio de fractura y fracturas vertebrales.

\section{Fuentes de datos y selección de estudios}

Se realizó una búsqueda sistemática de las publicaciones entre 1970 y Octubre de 2010 en MEDLINE, EMBASE y otras bases de datos. Diez publicaciones que reportaban 11 estudios fueron elegidos para desarrollar este meta análisis. Para poder ser elegidos, los estudios debían examinar el riesgo de fracturas atribuible al uso de inhibidores de la bomba de protones o antagonistas de los receptores histamínicos $\mathrm{H}_{2}$, y debían incluir un grupo control contra el cual comparar. El uso de la medicación se debía documentar antes que ocurriera la fractura y los análisis tenían que estar ajustados mínimamente por edad y sexo. No se filtraron artículos por idiomas.

\section{Resultados principales}

El riesgo de fractura de cadera aumentó modestamente entre los individuos consumidores de inhibidores de la bomba de pro-
Andrews RC y col. Lancet 2011;378:129-39. tones (RR 1,30; IC95\% 1,19 a 1,43). También se demostró un incremento en fracturas vertebrales (RR 1,56; IC95\% 1,31 a 1,85 ) y en cualquier sitio (RR 1,16; IC95\% 1,02 a 1,32). El consumo de antagonistas de los receptores histamínicos $\mathrm{H} 2$ no se asocio con aumento del riesgo de fracturas de cadera (RR 1,$12 ;$ IC $95 \% 0,97$ a 1,30 ) ni de fracturas en cualquier sitio (RR 0,99; IC95\% 0,86 a 1,15).

\section{Conclusiones}

Este meta análisis encontró que el uso de inhibidores de la bomba de protones producía un incremento discreto del riesgo de fracturas de cadera, fracturas vertebrales y fracturas en cualquier sitio, mientras que esta asociación no pudo ser demostrada con el uso de antagonistas de receptores histamínicos $\mathrm{H}_{2}$. El mecanismo biológico no está claro, por lo que no se puede descartar por completo el efecto de un posible confundidor. Hasta que no se obtengan datos más claros, los médicos deberíamos aconsejar a nuestros pacientes evitar la sobreutilización de inhibidores de la bomba de protones y considerar más evaluaciones en aquellos pacientes que consumen estos medicamentos y tienen alto riesgo de fracturas osteoporóticas.

Fuente de financiamiento: No referida.

\section{Comentario}

La osteoporosis es una condición muy prevalente en todo el mundo. Se estima una prevalencia del 3,5\% en mujeres entre los 50 y 54 años, y del $15 \%$ al $20 \%$ entre los 70 y 75 años de edad. En los últimos años, el tratamiento de la osteoporosis, y sobre todo la duración del mismo, han estado en constante revisión, ya que los beneficios del mismo a largo plazo no se han podido establecer con claridad. No obstante, la consecuencia más temida de la osteoporosis es la fractura de cadera, condición que genera una elevada morbimortalidad y alto costo en salud, ya sea por el tratamiento en si y por lo inherente a la rehabilitación y la perdida de autonomía que sufren los pacientes. Es por esto que pese a los beneficios poco establecidos del tratamiento con bifosfonatos en prevención primaria, estos se siguen indicando, sobre todo en población con mayor riesgo de fracturas. Debido a la intolerancia digestiva que frecuentemente ocasionan, habitualmente se los indica en conjunto con inhibidores de la bomba de protones (IBP), con el objetivo de aumentar la adherencia al tratamiento.
En este meta análisis, el uso de IBP se asoció con un incremento modesto del riesgo de fracturas de cadera (RR 1,30), vertebrales y fracturas en cualquier sitio, independientemente del genero y la duración del tratamiento, ya que se demostró esta asociación en tratamientos mayores y menores de 1 año de duración. Si los extrapolamos a la población general, en la que la sobreutilización de IBP constituye un verdadero problema, según los datos publicados en este artículo el $4,7 \%$ de las fracturas de cadera se podrían atribuir al uso de inhibidores de la bomba de protones.

Si bien son necesarios más estudios para poder establecer con claridad la relación entre fracturas óseas y uso de IBP, considero que estos datos nos tienen que servir en la práctica cotidiana para aconsejar a nuestros pacientes sobre el mal uso de estos medicamentos mundialmente difundidos (sobre todo en la población de mayor riesgo de fracturas), desestimando el uso de éstos cuando los beneficios no son claros.

Yamila Mahumud [ Servicio de Medicina Familiar y Comunitaria. Hospital Italiano de Buenos Aires. yamila.mahumud@ hospitalitaliano.org.ar]

Mahumud Y. El uso de Inhibidores de la bomba de protones puede aumentar el riesgo de fracturas óseas. Evid Act Pract Ambul. Oct-Dic 2012. 15(4).155. Comentado de: Yu EW, Bauer SR, Bain PA, et al. Proton Pump Inhibitors and Risk of Fractures: A Meta-Analysis of 11 International Studies. Am J Med. 2011;124(6):519-526. PMID:21605729. 